\title{
A fatal case of pneumococcal sepsis years after splenectomy
}

\author{
Kevin J. Solverson MD MSc, Christopher J. Doig MD MSc
}

Cite as: CMAJ 2017 June 12;189:E800-2. doi: 10.1503/cmaj.160455

A 42-year-old man developed sudden onset of fever, chills and generalized weakness one morning. This was preceded by several days of cough and sore throat. He contacted a provincial phone service staffed by registered nurses, who advised him on the treatment of "flu-like" illnesses. About six hours later, he presented to a regional urgent care centre.

As a child, the patient had a splenectomy for an unclear hematologic diagnosis. At age 26, he underwent allogenic liver transplantation for primary sclerosing cholangitis. Most recently, he had been treated with cyclosporine (50 $\mathrm{mg}$ orally twice daily) for chronic immunosuppression. He had regular follow-up and was considered well. He had received vaccination against infections postsplenectomy, including a booster pneumococcal vaccination (type unknown) within the two years before his current presentation. Peripheral blood smears taken before admission showed Howell-Jolly bodies consistent with asplenia.

At the time the patient was assessed in the urgent care centre, he was syncopal, with a blood pressure of $64 / 49 \mathrm{~mm} \mathrm{Hg}$, sinus tachycardia at 140 beats/minute, and a respiratory rate of 30 breaths/ minute. He was febrile at $39^{\circ} \mathrm{C}$. Because he was critically ill, he was transferred on an emergent basis to our tertiary care hospital.

In the emergency department, intravenous resuscitation was started, using large volumes of isotonic crystalloid; broadspectrum antibiotics were given. The patient required intravenous vasopressors for blood pressure support. A chest radiograph showed central peribronchial thickening and increased interstitial markings in both lower lobes. His serum lactate was elevated at 8.4 (normal $\leq 2.0$ ) $\mathrm{mmol} / \mathrm{L}$, and his serum $\mathrm{pH}$ was 6.95 (7.36-7.44). His white blood cell count was 3.2 (normal range 4.0$11.0) \times 10^{9} / \mathrm{L}$, with circulating premature leukocytes including $16 \%$ bands. He had multiple organ failure, including shock, respiratory failure, disseminated intravascular coagulation, hepatocellular dysfunction and renal failure.

The patient died about seven hours after presentation. Blood and sputum cultures grew Streptococcus pneumoniae (blood culture incubation positive at 5.4 hours) sensitive to penicillin. The patient's next of kin consented to postmortem examination, which confirmed the cause of death as septic shock from overwhelming postsplenectomy infection. Subsequent $S$. pneumoniae serotyping confirmed serotype $15 \mathrm{C}$.

\section{KEY POINTS}

- Patients who are asplenic should receive vaccinations for encapsulated organisms and other routine vaccinations, preferably before elective splenectomy.

- North American guidelines recommend vaccination against pneumococcus with the pneumococcal conjugate 13-valent and pneumococcal polysaccharide 23-valent vaccines in patients with asplenia.

- Patients should be educated that life-threatening infections are possible, and they should seek urgent assessment if they develop any signs of infection, specifically a fever.

- A history of splenectomy or evidence of hyposplenism (e.g., Howell-Jolly bodies on a peripheral smear) in a patient with fever or infection may indicate a life-threatening condition and requires immediate cultures and prompt initiation of antibiotic therapy.

\section{Discussion}

This case illustrates the risk of fulminant sepsis and death in patients who have undergone prior splenectomy. Asplenia (surgical splenectomy) or hyposplenism secondary to an underlying medical condition is common. An estimated prevalence of asplenia in an Eastern Canadian cohort was 1 per 1000 persons. ${ }^{1}$ In this cohort, $48.6 \%$ of patients had a splenectomy for a preexisting hematologic diagnosis, such as immune thrombocytopenic purpura. Splenic rupture from trauma has been a common indication for splenectomy; however, with the advent of therapies such as endovascular embolization, the frequency of splenectomy in trauma is decreasing. ${ }^{2}$

The spleen plays an important role in the body's immune defence from invasive infection, particularly from encapsulated bacteria (S. pneumoniae, Haemophilus influenzae and Neisseria meningitidis). Invasive infections have been reported to occur in $3.2 \%$ of asplenic or hyposplenic patients and $1.4 \%$ of all patients die from infection. ${ }^{3}$ If a patient progresses to overwhelming postsplenectomy infection, which includes septic shock and multiorgan failure, the mortality rate can reach $70 \%$, which is substantially higher than the mortality rate of general medical patients with severe sepsis. ${ }^{2,4}$ 


\section{Vaccinations}

The rate of infection is thought to be highest for the first three years after splenectomy, but it remains elevated for decades. $S$. pneumoniae is the most clinically significant pathogen, accounting for between $50 \%$ and $90 \%$ of cases of overwhelming postsplenectomy infection. ${ }^{2}$ The spleen has specific macrophages capable of clearing S. pneumoniae and memory B cells that produce natural antibodies against these organisms. The loss of these cells is thought to make asplenic patients more susceptible to invasive pneumococcal infection.

The increased prevalence and mortality from invasive infections has led to the development of immunization guidelines for asplenic patients. The current Canadian, British and American immunization guidelines recommend that all asplenic and hyposplenic patients receive $H$. influenzae, N. meningitidis, S. pneumoniae, influenza and other indicated routine vaccines (Box 1). ${ }^{5,6}$ Patients undergoing elective splenectomy should receive all the vaccines two weeks before surgery and, in the case of an emergency splenectomy, vaccines should be given two weeks postsplenectomy. ${ }^{5}$

Historically, vaccine rates have been low in asplenic patients, despite the known dangers of bacterial infections. In a cohort of Canadian asplenic patients, only $16.5 \%$ were vaccinated against S. pneumoniae, although a recent 2015 report from the United States showed that $81 \%$ of patients had received the vaccine., Until recently, the primary pneumococcal vaccination was a polysaccharide 23-valent vaccine (Pneu-P-23), which covers 23 prevalent serotypes. The vaccine relies on a $\mathrm{T}$ cell-independent immune response, which may be impaired in asplenic patients, due to a lack of native splenic B cells. Recently, pneumococcal conjugated vaccines have been created that induce a $\mathrm{T}$ celldependent immune response and an immunologic memory. This

Box 1: Immunization and prevention strategies against infection in asplenic and hyposplenic patients

\section{Canadian immunization recommendations for patients with asplenia or hyposplenia*}

Pneumococcal polysaccharide 23-valent and pneumococcal conjugate 13 -valent vaccines

Haemophilus influenzae type b conjugate vaccine

Quadrivalent conjugate meningococcal or multicomponent meningococcal vaccine

Any other routine vaccinations that are indicated

\section{Other preventive} considerations $^{13}$

Educate patients to seek immediate medical assessment in case of fever or acute medical illness and to consider wearing a medical-alert tag

Consider lifelong antibiotic prophylaxis

Provide access to standby antibiotics at home in case of fever or acute infection symptoms

Advise patients to seek medical because of infectious risk from malaria and animal bites assessment before travel

*See the Canadian Immunization Guide for timing and dosing of vaccines: http:// healthycanadians.gc.ca/publications/healthy-living-vie-saine/3-canadian-immunization -guide-canadien-immunisation/index-eng.php?page $=7$. type of vaccine has shown to increase immunogenicity in asplenic patients when added to the Pneu-P-23 vaccine. ${ }^{7,8}$

In 2013, guidelines published by the Canadian National Advisory Committee on Immunizations and the Infectious Diseases Society of America recommended that asplenic patients receive the pneumococcal 13-valent conjugate vaccine (Pneu-C-13) once, in addition to the Pneu-P-23 vaccine (administered eight weeks after the conjugate vaccine, and a booster given five years later). ${ }^{6,9}$ Since the implementation of these recommendations, the rate of invasive pneumococcal disease in Canada has been stable, at about 9.6 cases per $100000 .{ }^{10}$ There has been a decrease in the incidence of pneumococcal serotypes covered by Pneu-C-13, but also a possible increase in serotypes not covered by the pneumococcal vaccines, specifically in the asplenic population. ${ }^{10,11}$ The current Canadian vaccine guidelines can be found online at www. phac-aspc.gc.ca/publicat/cig-gci/index-eng.php.

\section{Antibiotic prophylaxis}

Despite vaccination, patients can still be susceptible to bacterial infections, which has led to the development of additional recommendations. Antibiotic prophylaxis for encapsulated bacteria is controversial in the literature. The Canadian Paediatric Society recommends antibiotic prophylaxis for asplenic patients at least until the age of five years. ${ }^{12}$ The society and other experts recommend that lifelong antibiotic prophylaxis be considered for all patients with asplenia, especially if the patient has had a prior pneumococcal infection or has a concurrent immune deficiency. ${ }^{12,13}$ However, concerns over compliance and the development of resistant organisms have been raised. ${ }^{13}$

\section{Early initiation of antibiotics}

When an asplenic patient develops a bacterial infection, starting broad-spectrum antibiotics early is the foundation to preventing further morbidity and mortality. In all patients with infections associated with severe sepsis, early use of antibiotics is associated with improved outcomes and is standard of care. ${ }^{14}$

Many experts recommend that asplenic patients have standby antibiotics at home and be instructed to take a dose if they develop a fever or symptoms of infection, and then to present to a medical facility..$^{13}$ It is crucial to educate patients so that they understand their risk of life-threatening infection and know the proper actions to take if symptoms of an infection develop. Unfortunately, studies have shown that many patients do not understand their risk of severe infection or know what to do if they become unwell. ${ }^{2}$ Experts also suggest that patients be given written instruction on the actions to take and who to contact in the event of symptoms of infection and also that they wear a medical-alert bracelet. ${ }^{13}$

\section{Conclusion}

With respect to our case, there are some important points that highlight the above discussion. Despite the patient receiving the pneumococcal vaccine and a booster, he developed septic shock secondary to bacteremia with S. pneumoniae, serotype $15 \mathrm{C}$. This serotype is not contained in the current conjugated or polysaccharide vaccines. Therefore, this patient's death was not preventable by vaccination. However, conjugated vaccines do improve 
immunogenicity in asplenic patients, creating better protection from invasive pneumococcal infections, and thus are recommended by guidelines.

The family does not think that the patient was aware of antibiotic prophylaxis or standby antibiotics for fever. Although there is controversy about lifelong antibiotic prophylaxis, most experts suggest that patients have standby antibiotics at home in the event of a fever.

Additionally, physicians and other health care professionals should consider starting antibiotics early if an asplenic patient develops fever. Perhaps most important is the need to provide these patients with an action plan if they develop fever, including reminding health care professionals of their asplenia.

\section{References}

1. Langley JM, Dodds L, Fell D, et al. Pneumococcal and influenza immunization in asplenic persons: a retrospective population-based cohort study 1990-2002. BMC Infect Dis 2010;10:219.

2. Di Sabatino A, Carsetti R, Corazza GR. Post-splenectomy and hyposplenic states. Lancet 2011;378:86-97.

3. Bisharat N, Omari H, Lavi I, et al. Risk of infection and death among post-splenectomy patients. J Infect 2001;43:182-6.

4. Kaukonen KM, Bailey M, Suzuki S, et al. Mortality related to severe sepsis and septic shock among critically ill patients in Australia and New Zealand, 20002012. JAMA 2014;311:1308-16.
5. Canadian immunization guide: Part 3 -Vaccination of specific populations Ottawa: Public Health Agency of Canada; 2014.

6. Rubin LG, Levin MJ, Ljungman P, et al.; Infectious Diseases Society of America. 2013 IDSA clinical practice guideline for vaccination of the immunocompromised host. Clin Infect Dis 2014;58:309-18.

7. Nived $P$, Jørgensen CS, Settergren B. Vaccination status and immune response to 13 -valent pneumococcal conjugate vaccine in asplenic individuals. Vaccine 2015;33:1688-94.

8. Forstner C, Plefka S, Tobudic S, et al. Effectiveness and immunogenicity of pneumococcal vaccination in splenectomized and functionally asplenic patients. Vaccine 2012;30:5449-52.

9. Statement on the use of conjugate pneumococcal vaccine -13 valent in adults (Pneu-C-13). Can Commun Dis Rep 2013;39(ACS-5):1-52. Available: www. phac-aspc.gc.ca/publicat/ccdr-rmtc/13vol39/acs-dcc-5/index-eng.php (accessed 2017 Feb. 8).

10. Teatero S, Ferrieri P, Martin I, et al. Serotype distribution, population structure and antimicrobial resistance of Group B Streptococcus strains recovered from colonized pregnant women. J Clin Microbiol 2017;55:412-22.

11. Marrie TJ, Tyrrell GJ, Majumdar SR, et al. Asplenic patients and invasive pneumococcal disease-how bad is it these days? Int J Infect Dis 2016;51:27-30.

12. Salvadori MI, Price VE; Canadian Paediatric Society, Infectious Diseases and Immunization Committee. Preventing and treating infections in children with asplenia or hyposplenia. Paediatr Child Health 2014;19:271-8.

13. Davies JM, Lewis MP, Wimperis J, et al.; British Committee for Standards in Haematology. Review of guidelines for the prevention and treatment of infection in patients with an absent or dysfunctional spleen: prepared on behalf of the British Committee for Standards in Haematology by a working party of the Haemato-Oncology task force. Br J Haematol 2011;155:308-17.

14. Kumar A, Roberts D, Wood KE, et al. Duration of hypotension before initiation of effective antimicrobial therapy is the critical determinant of survival in human septic shock. Crit Care Med 2006;34:1589-96.

\section{Competing interests: None declared.}

This article has been peer reviewed.

The authors have obtained patient consent.

Affiliations: Departments of Critical Care Medicine (Solverson, Doig) and Community Health Sciences (Doig), Cumming School of Medicine, University of Calgary, Calgary, Alta.
Contributors: Kevin Solverson and Christopher Doig provided equal input into the creation and critical revision of the manuscript. Both authors approved the final version to be published, and agreed to act as guarantors of the accuracy of the work.
Acknowledgement: The authors would like to thank Dr. James Kellner, from the Alberta Children's Hospital, for his thoughtful comments and review of the manuscript.

Correspondence to: Christopher Doig, cdoig@ucalgary.ca 